\title{
The effect of brief pre-anesthetic exercise therapy of jaw and neck joints on mouth opening, neck extension, and intubation conditions during induction of general anesthesia: a randomized controlled trial
}

Sue Young Lee ${ }^{1 \dagger}$, Sung II Bae ${ }^{2 \dagger}$, Sang-Hwan Do ${ }^{3,4}$, Ju-Tae Sohn ${ }^{2,5}$ and Jin-Woo Park ${ }^{3^{*}}$ (D)

\begin{abstract}
Background: The effort to improve tracheal intubation process is clinically valuable. We hypothesized that a preoperative brief exercise therapy would increase mouth opening and neck extension, enhancing intubation conditions during general anesthesia.

Methods: Patients undergoing general anesthesia were randomized into two groups. The exercise group performed the exercise regimen including masseter muscle massage and stretching of jaw and neck joints before anesthetic induction, while the control did not. Before (baseline) and after the intervention, we evaluated Mallampati score, mouth aperture size, and sternomental distance. After tracheal intubation, intubation difficulty scale with direct laryngoscope and oropharyngeal soft tissue injury were also evaluated.

Results: A total of 138 patients completed the analysis (control $=68$, exercise $=70$ ). Baseline characteristics did not differ between groups. At anesthetic induction, there was a significant difference in Mallampati score between the two groups ( $P=0.039$ ) and the incidence of Mallampati scores of 1 was higher in the exercise group (odds ratio [95\% Cl]: $2.1[1.0-4.3], P=0.043)$. Mouth opening after the intervention was greater in the exercise group than in the control group (estimated difference [95\% Cl]: $-2.4[-4.8--0.1], P=0.042$ ) and sternomental distance was similar between the two groups (estimated difference [95\% Cl]: $-3.7[-9.0-1.7, P=0.175$ ). The exercise group showed less soft tissue injuries (odds ratio [95\% Cl]: 0.2 [0.1-0.8], $P=0.009$ ), however, intubation difficulty scale did not differ between the study groups $(P=0.112)$.
\end{abstract}

Conclusions: The brief pre-anesthetic exercise improved intubation conditions and enabled faster tracheal intubation with less injury to oropharyngeal soft tissue.

Trial registration: Clinical Research Information Service (registration number: KCT0002618), registered at December 28, 2017.

Keywords: Anesthesia, general, Exercise, Intubation, Mouth opening, Neck extension

\footnotetext{
* Correspondence: jinul8282@gmail.com

+ Sue Young Lee and Sung II Bae contributed equally to this work.

${ }^{3}$ Department of Anesthesiology and Pain Medicine, Seoul National University

Bundang Hospital, 82 Gumi-ro, 173 Beon-gil, Bundang-gu, Seongnam,

Gyeonggi-do 13620, Republic of Korea

Full list of author information is available at the end of the article
}

(c) The Author(s). 2020 Open Access This article is distributed under the terms of the Creative Commons Attribution 4.0 International License (http://creativecommons.org/licenses/by/4.0/), which permits unrestricted use, distribution, and reproduction in any medium, provided you give appropriate credit to the original author(s) and the source, provide a link to the Creative Commons license, and indicate if changes were made. The Creative Commons Public Domain Dedication waiver (http://creativecommons.org/publicdomain/zero/1.0/) applies to the data made available in this article, unless otherwise stated. 


\section{Background}

Limited neck movement and a short inter-incisor gap may interfere with exposure of the larynx during direct laryngoscopy and are significant risk factors for difficult tracheal intubation [1-5]. When glottic exposure is inadequate with a direct laryngoscope, other instruments such as intubation stylet, video laryngoscope, or fiberoptic bronchoscope can be utilized to access the larynx $[6,7]$. However, difficult intubation cannot be exactly predicted in advance $[3,5,8]$. Complications such as soft tissue injuries and dental trauma may also occur during successful intubation $[9,10]$. Without a doubt, improvement of intubation conditions is of clinical significance for patients undergoing general anesthesia.

Various exercise regimens based on stretching and muscle massage have been proposed to treat patients with temporomandibular joint disorders and impaired mouth opening [11]. Furthermore, previous studies demonstrated that brief stretching or massage of joint muscles could increase range of motion and decrease muscle stiffness in healthy volunteers as well as subjects with musculoskeletal abnormalities [12-18]. Therefore, we hypothesized that pre-anesthetic exercise therapy of jaw (temporomandibular) and neck (cervical vertebral) joints could enhance mouth opening and neck mobility during anesthetic induction and facilitate the process of orotracheal intubation.

We performed a randomized controlled trial to evaluate the effects of a single brief pre-anesthetic exercise therapy session on mouth opening, neck extension, intubation difficulty, and soft tissue injuries to the oropharyngeal area in patients undergoing general anesthesia.

\section{Methods}

\section{Study}

The protocol for this prospective multicenter trial was approved by the Institutional Review Boards of Seoul National University Bundang Hospital (study number: B1709-423-307) and Jinju Gyeongsang National University Hospital (study number: GNUH 2018-05-019-001). It was registered at the Clinical Research Information Service (https://cris.nih.go.kr; registration number: KCT0002618; date of registration: December 28, 2017). Written informed consent was obtained from all patients before surgery. Patients were enrolled from January to October 2018 at both institutions. This study adhered to CONSORT guidelines.

\section{Patients}

Adult patients with American Society of Anesthesiologists physical status I-II, aged 20-70 years, who were scheduled to undergo elective surgery under general anesthesia were enrolled in this study. We excluded patients who were likely to encounter difficulties or pain when actively performing the jaw and neck exercises: Patients with cognitive disorders, temporomandibular joint disorders, or cervical spine diseases. For safety reasons, we excluded patients under consideration for awake intubation using a fiber-optic bronchoscope, such as those with craniofacial anomaly or mechanical airway obstruction. Patients with tracheostomy or who were to receive oropharyngeal surgery during this protocol were also excluded. Enrolled patients were randomly allocated to either the exercise or the control group, using a computer-generated randomization code (Random Allocation Software Version 1.0; University of Medical Sciences, Isfahan, Iran). The allocation ratio was 1:1. Randomization was performed by an independent anesthesiologist in each hospital. These individuals were only involved in patient allocation and coaching of the exercise, and not in any aspects of anesthesia, data analysis or interpretation.

\section{Intervention, anesthesia, and study outcomes}

At the reception area, each patient's basal characteristics related to tracheal intubation difficulty were assessed [2-5]. These included mouth aperture size defined as the maximum interincisal distance, Mallampati score, sternomental distance (SMD) as an indicator of neck mobility, thyromental distance, and the presence of buck teeth, were assessed by an anesthesiology resident in each hospital who only participated in these examinations and did not know the patient group. We used a single assessor in each hospital, to minimize inter-rater bias. In this protocol, Mallampati scoring was performed in sitting position, with the tongue fully protruded, and without phonation [19]. During the measurements, the sitting patients were encouraged to open their mouths as wide as possible and to fully extend their head on the neck. After the baseline examination, patients in the exercise group were guided to perform exercise therapy for $5 \mathrm{~min}$ at the reception area, before transferral to the operating room. This brief exercise regimen comprises masseter muscle massage and active/passive stretching of the jaw and neck joints (Fig. 1). Stretching was performed to the maximum range of motion within the level not causing pain. The patients in the control group waited at the reception area, without any exercise, before transfer to the operating room. No pre-medication was given to patients in either group. Patients were instructed not to reveal their group information to anyone after the intervention. The importance of blindness was emphasized repeatedly to all patients throughout the protocol.

After transport to the operating room, standard non-invasive monitoring of vital signs was started. 


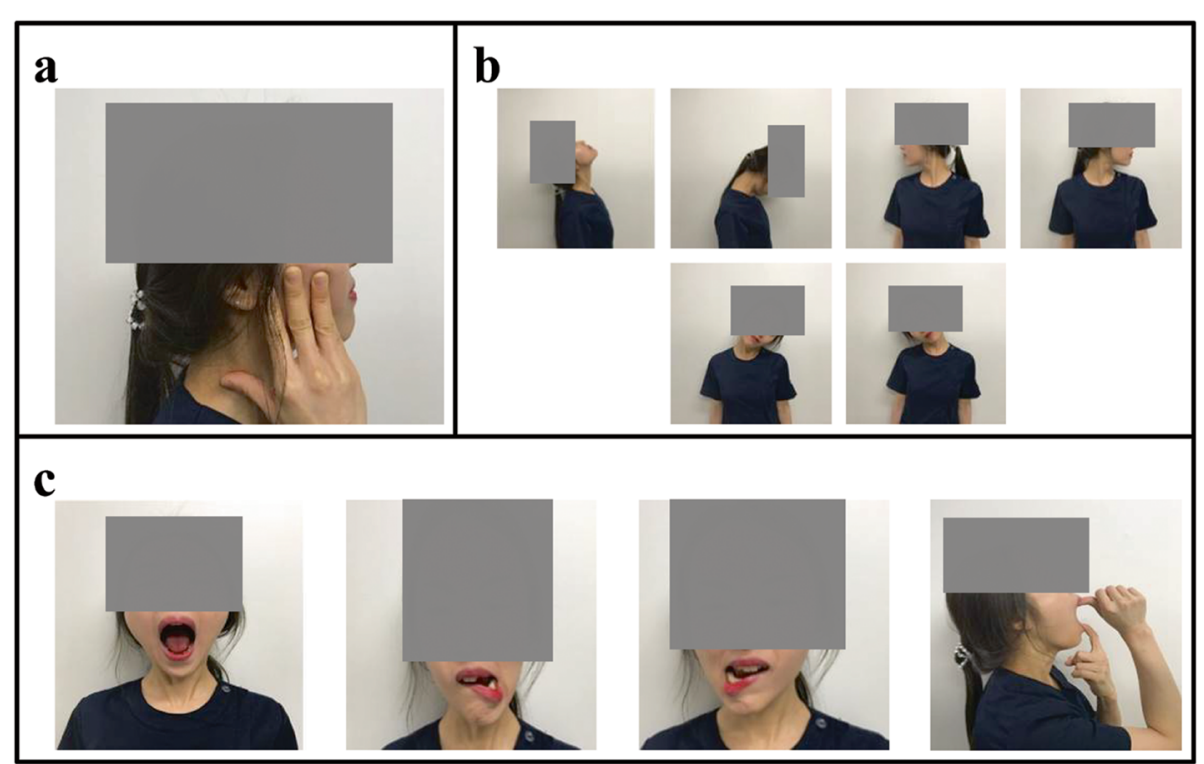

Fig. 1 The exercise regimen on jaw and neck joints (Exercise sequence: $a-b-c-a)$. a Manual massage of masseter muscle (30 s); $\mathbf{b}$ Extension, flexion, rotation, and lateral flexion of neck ( $2 \mathrm{~min})$; c Active and passive maximum mandibular opening and lateral deviation of temoporomandibular joint (2 min)

Mouth aperture, Mallampati score, and SMD were measured again, just before anesthetic induction. In each hospital, the induction of general anesthesia and intubation were performed by a blinded anesthesiologist (clinical professor) who was unaware of patient allocation, and had more than 7 years of experience with anesthesia. After denitrogenation with $100 \%$ oxygen, propofol 1.5-2 $\mathrm{mg} / \mathrm{kg}$ was intravenously administered, and targetcontrolled infusion of remifentanil with $4 \mathrm{ng} / \mathrm{ml}$ effect site concentration was started to inhibit hemodynamic responses to tracheal intubation. After patient consciousness and eyelash reflex were lost, rocuronium $0.6 \mathrm{mg} / \mathrm{kg}$ was administered, and facial mask ventilation was performed. Muscle relaxation was assessed using train-of-four stimuli every $10 \mathrm{~s}$. Following the disappearance of $\mathrm{T} 1$ of train-offour stimuli, orotracheal intubation was performed using a direct laryngoscope with Macintosh blade. During intubation, the anesthesiologist placed the laryngoscope into the mouth and raised the blade up and away from the patient to obtain a clear view of the glottis. The subjective necessity of increased lifting force compared to routine practice, and the Cormack-Lehane grade, were checked. To facilitate intubation, laryngeal compression (backward, upward, and rightward pressure) was sometimes applied by an assistant [20]. If we could not obtain an adequate view, orotracheal intubation was attempted with alternative techniques including intubation stylet, video laryngoscope, and/or fiber-optic bronchoscope. If the first anesthesiologist failed to intubate within 3 attempts, the fourth trial was performed by another senior anesthesiologist [21]. The requirements for laryngeal compression or alternative techniques, the number of intubation attempts and additional anesthesiologists, and the intubation time from the end of mask ventilation to the passage of an orotracheal tube through the vocal cords, were recorded by an independent and blinded anesthetic nurse in each hospital. Intubation difficulty scale (IDS) was calculated based on these values (Fig. 2) $[2,22,23]$.

After anesthetic induction, anesthetic depth was maintained using 1-1.5 minimum alveolar concentration inhalation (sevoflurane or desfluane), or targetcontrolled infusion of propofol at a 40-60 bispectral index, with remifentanil target-controlled infusion adjusted to maintain hemodynamic stability. At the end of the operation, neostigmine $0.03 \mathrm{mg} / \mathrm{kg}$ and glycopyrrolate $0.01 \mathrm{mg} / \mathrm{kg}$ were administered to reverse residual neuromuscular block. When the train-of-four ratio increased over $90 \%$ and sufficient spontaneous breathing was confirmed, oropharyngeal secretions were gently cleared by suction, and the endotracheal tube removed.

At the post anesthesia care unit of each hospital, 15 min after the extubation, injuries of oropharyngeal soft tissue including lip, gum, tongue, buccal mucosa, palate, and pharynx were examined using a penlight by a single blinded anesthesiologist who participated only in this measurement and had no other involvement in the study. Except in nasal surgery cases, the appearance of bloody secretions in the oropharyngeal suction before extubation was also interpreted as incidence of oropharyngeal injury. 


\section{Intubation Difficulty Scale (IDS)}

\begin{tabular}{|c|c|}
\hline Parameter & Score \\
\hline Number of Attempts $>1$ & $\mathbf{N}_{1}$ \\
\hline Number of Operators $>1$ & $\mathbf{N}_{2}$ \\
\hline $\begin{array}{l}\text { Number of Alternative } \\
\text { techniques }\end{array}$ & $\mathbf{N}_{3}$ \\
\hline Cormack and Lehane Grade - 1 & $\mathbf{N}_{4}$ \\
\hline $\begin{array}{l}\text { Lifting Force Required } \begin{array}{r}\text { Normal } \\
\text { Increased }\end{array}\end{array}$ & $\begin{array}{l}\mathrm{N}_{5}=0 \\
\mathrm{~N}_{5}=1\end{array}$ \\
\hline $\begin{array}{l}\text { Cricoid Compression } \\
\qquad \text { Not applied } \\
\text { Applied }\end{array}$ & $\begin{array}{l}N_{6}=0 \\
N_{6}=1\end{array}$ \\
\hline $\begin{array}{ll}\text { Vocal cord Mobility } & \\
& \text { Abduction } \\
\text { Adduction }\end{array}$ & $\begin{array}{l}N_{7}=0 \\
N_{7}=1\end{array}$ \\
\hline $\begin{array}{l}\text { TOTAL: IDS = SUM OF } \\
\text { SCORES }\end{array}$ & $\mathbf{N}_{1}-\mathbf{N}_{7}$ \\
\hline
\end{tabular}

Fig. 2 Intubation difficulty scale

\begin{tabular}{|l|l|}
\hline \multicolumn{1}{|c|}{ Score } & \multicolumn{1}{c|}{ Rule } \\
\hline $\mathbf{N}_{1}$ & Every additional attempt adds 1 pt. \\
\hline $\mathbf{N}_{2}$ & Each additional operator adds 1 pt. \\
\hline $\mathbf{N}_{3}$ & $\begin{array}{l}\text { Each alternative technique adds 1 point: } \\
\text { intubation stylet, video laryngoscope, and/or } \\
\text { fiber-optic bronchoscope. }\end{array}$ \\
\hline $\mathbf{N}_{4}$ & Apply Cormack grade for 1 1 st oral attempt. \\
\hline IDS Score & \multicolumn{1}{|c|}{ Degree of Difficulty } \\
\hline 0 & Easy \\
\hline $0<$ IDS $\leq 5$ & Slight Difficulty \\
\hline $5<$ IDS & Moderate to Major Difficulty \\
\hline
\end{tabular}

\section{Statistical analysis}

Continuous variables are expressed as mean (SD). Categorical variables are shown as number or percentage (\%). The primary outcome was the Mallampati score before and after exercise. Secondary outcomes were as follows: mouth aperture size, SMD, Cormack-Lehane grade, intubation time, numbers of intubation attempts, number of anesthesiologists who attempted orotracheal intubation, number of alternative techniques required, increased lifting pressure or laryngeal compression, soft tissue injuries after using direct laryngoscope, and IDS. To compare continuous variables between control and exercise groups, we used the independent Student's ttest. Differences between categorical variables were assessed using the chi-square or Fisher's exact test between the two groups. For grading characteristic of Mallampati score and Cormack-Lehane grade, linear-bylinear association was performed to compare the trend between groups. To analyze within-group change between before and after the exercise therapy, a paired $t$ test was used. SPSS version 19.0 software (SPSS Inc., IBM, Chicago, IL, USA) was used for the statistical analysis. All reported $P$-values are two-sided, and a $P$-value $<0.05$ was considered statistically significant.

\section{Sample size}

In a pilot study of 20 patients (10 patients in each group), the effect size for Mallampati score was used to calculate the required sample size (number of patients). The Mallampati score indicates mouth aperture size relative to tongue size, and therefore reflects the adequacy of mouth opening during displacement of the tongue by the laryngoscope $[3,19,24]$. Mallampati score is one of the well-recognized predictive factors for the condition of orotracheal intubation, and a Mallampati score other than 1 was reported as one of the criteria for predicting the condition [3,5]. The incidence of Mallampati scores of 1 after the exercise regimen were $15 \%$ for the control group, and 35\% for the exercise group. Based on the results of the pilot study, a power analysis was performed with $G^{*}$ Power 3.1.2 (Heinrich-Heine University, Düsseldorf, Germany). This suggested that in each group, 70 patients were required for a power of $80 \%$, a risk of 0.05 for a type-I error in two-tailed statistical analysis, and a dropout rate of $7.5 \%$.

\section{Results}

Of the 151 eligible patients, 11 were excluded because 8 declined to participate, and 3 met the exclusion criteria 
for cervical pain. The remaining 140 patients were randomly allocated to one of the two study groups, with 70 patients per group. Two patients in the control group withdrew after the training intervention. Thus, 138 patients completed the study (number of patients in the control and exercise groups, 44 and 47 respectively in Seoul National University Bundang Hospital, and 24 and 23 in Jinju Gyeongsang National University Hospital; Fig. 3).

Patients' baseline characteristics did not differ between groups, including mouth aperture size, SMD, preintervention Mallampati score, anesthesia, and surgery data affecting the intubation difficulty or the postoperative soft tissue assessment between the study groups (Tables 1 and 2).

At anesthetic induction, there was a significant difference in Mallampati score between the two groups $(P=$ 0.039 ) and the incidence of Mallampati scores of 1 was higher in the exercise than in the control group (2.1 (1.0-4.3), odds ratio $(95 \% \mathrm{CI}) ; P=0.043$; Table 2$)$. Mouth opening was after the intervention greater in the exercise group than in the control group $(P=0.042)$, however, SMD was indistinguishable between the control and exercise groups $(P=0.175$; Table 2$)$. Analysis of pre- to post-exercise therapy changes within the exercise group showed that the exercise intervention increased mouth aperture size and SMD $(P<0.001$; Table 2$)$.
The incidences of increased lifting force and laryngeal compression required to obtain the intubation pathway were lower in the exercise than in the control group $(P=0.034$ and 0.027 , respectively; Table 3$)$. All other variables of IDS, the IDS score itself, and IDS difficulty group did not differ significantly between the two groups (Table 3). In both groups, there was no case in which an additional anesthesiologist was requested to attempt tracheal intubation (Table 3). Compared to the control group, the exercise group showed shorter intubation time and fewer soft tissue injuries $(P=0.032$ and 0.009 , respectively; Table 3 ). There were 58 patients with a baseline Mallampati score III or IV, who were deemed to show smaller mouth opening during tracheal intubation. In the subgroup analysis performed for those patients, there was a significant difference in the IDS grade between the control and exercise groups $(P=0.029$; Table 4). The Cormack-Lehane grade did not significantly differ between the groups in the subgroup comparison (Table 4).

\section{Discussion}

The beneficial effects of stretching or massage on different joints have been studied for various therapeutic applications [12-18]. A variety of exercise-treatments have been demonstrated to relieve clinical symptoms and restore range of motion in joint disorders, including

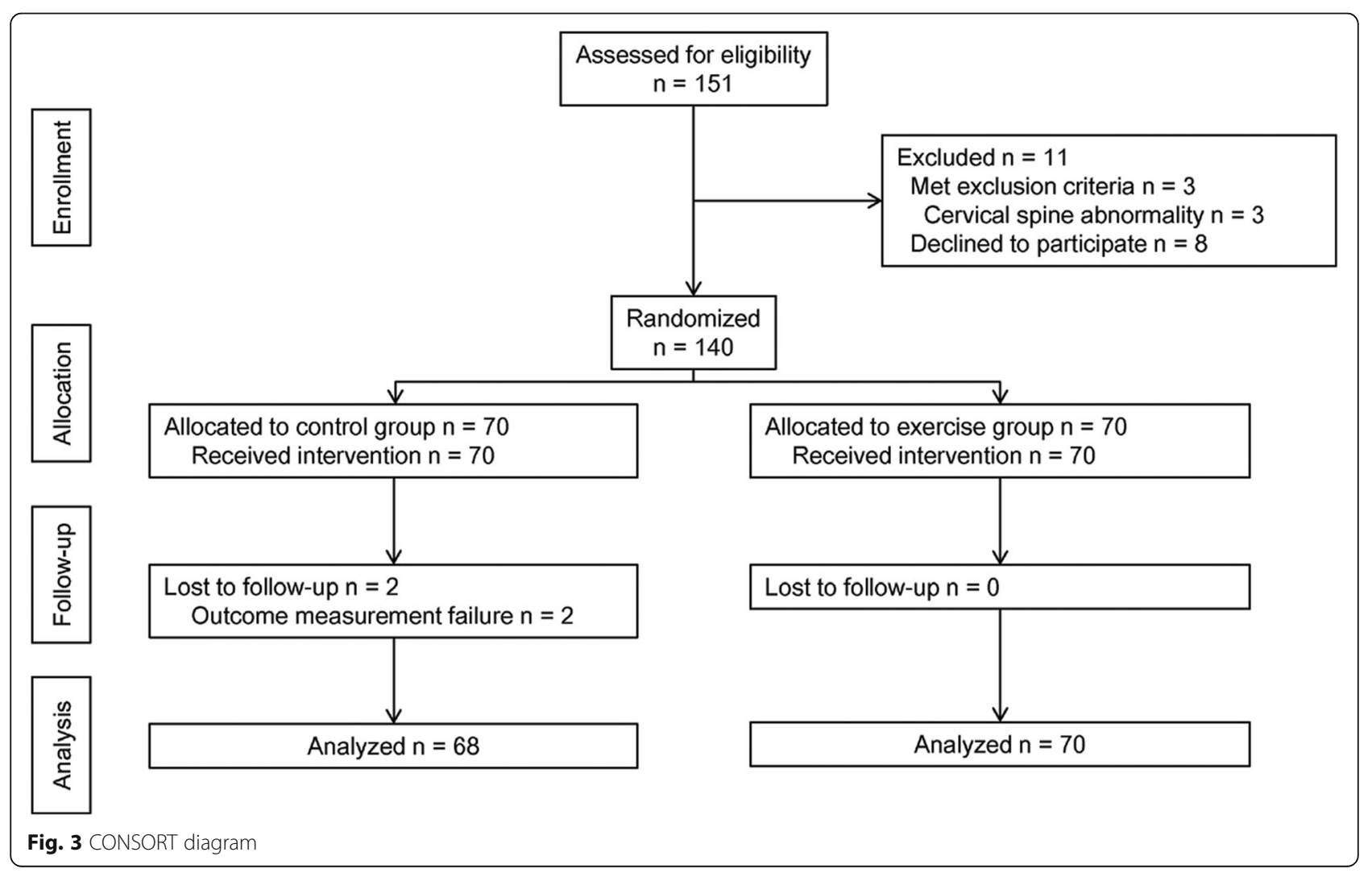


Table 1 Patients' characteristics, anesthesia, and operational data

\begin{tabular}{lll}
\hline & Control $(n=68)$ & Exercise $(n=70)$ \\
\hline Age (years) & $49.5 \pm 11.6$ & $50.6 \pm 10.6$ \\
Male sex & $32(47.1 \%)$ & $32(45.7 \%)$ \\
Height (cm) & $165.1 \pm 9.1$ & $163.9 \pm 9.0$ \\
Weight (kg) & $65.2 \pm 11.8$ & $68.0 \pm 11.4$ \\
ASA class (I/I) & $27(39.7 \%) / 41(60.3 \%)$ & $23(32.9 \%) / 47(67.1 \%)$ \\
Thyromental distance (mm) & $91.1 \pm 9.8$ & $91.8 \pm 10.2$ \\
Buck teeth & $1(1.5 \%)$ & $3(4.3 \%)$ \\
Induction dose (propofol, mg) & $112.6 \pm 13.2$ & $114.9 \pm 10.5$ \\
Anesthetic time (min) & $123.6 \pm 74.7$ & $139.9 \pm 82.4$ \\
Operation Position (supine/prone/lateral decubitus) & $66 / 2 / 0$ & $69 / 0 / 1$ \\
Nasal surgery & $4(5.9 \%)$ & $9(12.9 \%)$
\end{tabular}

Continuous values are shown as mean \pm SD. Categorical variables are expressed as patient numbers (\%) or numbers

ASA American society of anesthesiologists

osteoarthritis and rheumatoid arthritis [11, 25-28]. The exercise regimen of our study protocol included active and passive stretching of jaw and neck muscles. A brief stretching session potentially enhances joint flexibility and alleviates muscle stiffness $[12,13,18]$. In a previous study of knee joint exercise, 4 sets of stretching for $20 \mathrm{~s}$ each produced a decrease in passive hamstring stiffness, and improved joint range of motion [12]. Massage has been also reported to reduce muscle stiffness $[15,16]$. In our protocol, the patients in the exercise group performed masseter muscle massage at the beginning and at the end of the exercise therapy. To our knowledge, this study is the first quantification of the effect of a simple exercise regimen on mouth opening and neck extension during anesthetic induction.

During induction of general anesthesia, orotracheal intubation is usually performed under adequate muscle relaxation that can be achieved by administration of a neuromuscular blocking agent. However, a neuromuscular blocking agent exerts its effects at the neuromuscular junction. It does not modulate the mechanical properties of joints but rather interferes with neural drive to muscle, thereby blocking contraction [29]. Joint flexibility or stiffness is dependent not only upon the contraction of joint muscles, but also on the properties of connective tissue and ligaments [30-33]. Therefore, the enhancement of mouth opening and neck mobility by pre-anesthetic exercise could be maintained even after neuromuscular blocking agent injection. Furthermore, it was reported that general anesthesia employing muscle relaxants did not necessarily increase the passive range of motion of joints [30].

Although SMD examined just before anesthetic induction was comparable between the two groups, the clear differences before and after the regimen within the exercise group showed the definite beneficial effects of our exercise therapy on mouth opening and neck extension. The exercise group showed larger mouth opening and

Table 2 Mouth aperture size, SMD, and Mallampati score

\begin{tabular}{|c|c|c|c|c|c|c|c|c|c|c|c|}
\hline & \multicolumn{2}{|c|}{ Control $(n=68)$} & \multicolumn{2}{|c|}{ Exercise $(n=70)$} & \multirow[t]{2}{*}{$P_{1}^{a}$} & \multirow[t]{2}{*}{$P_{2}^{b}$} & \multirow{2}{*}{$\begin{array}{l}\text { Estimated } \\
\text { difference }{ }^{\mathrm{b}}(95 \% \\
\mathrm{Cl})\end{array}$} & \multirow[t]{2}{*}{$P_{3}{ }^{c}$} & \multirow{2}{*}{$\begin{array}{l}\text { Odds } \\
\text { ratio }^{c}(95 \% \mathrm{Cl})\end{array}$} & \multirow[t]{2}{*}{$P_{4}^{d}$} & \multirow{2}{*}{$\begin{array}{l}\text { Estimated } \\
\text { difference }^{d}(95 \% \\
\text { Cl) }\end{array}$} \\
\hline & Before & After & Before & After & & & & & & & \\
\hline $\begin{array}{l}\text { Mouth aperture size } \\
(\mathrm{mm})\end{array}$ & $\begin{array}{l}49.3 \pm \\
6.4\end{array}$ & $\begin{array}{l}49.0 \pm \\
7.5\end{array}$ & $\begin{array}{l}48.9 \pm \\
5.0\end{array}$ & $\begin{array}{l}51.3 \pm \\
6.4\end{array}$ & 0.675 & 0.042 & $-2.4(-4.8$ to -0.1$)$ & & & $<0.001$ & $-2.5(-3.7$ to -1.2$)$ \\
\hline $\mathrm{SMD}(\mathrm{mm})$ & $\begin{array}{l}183.6 \pm \\
16.0\end{array}$ & $\begin{array}{l}183.6 \pm \\
16.0\end{array}$ & $\begin{array}{l}181.8 \pm \\
16.0\end{array}$ & $\begin{array}{l}187.3 \pm \\
15.8\end{array}$ & 0.505 & 0.175 & $-3.7(-9.0$ to 1.7$)$ & & & $<0.001$ & $-5.5(-6.4$ to -4.6$)$ \\
\hline $\begin{array}{l}\text { Mallampati score (I/ } \\
\text { II/II/IV) }\end{array}$ & $\begin{array}{l}18 / 22 / \\
14 / 14\end{array}$ & $\begin{array}{l}18 / 23 / \\
14 / 13\end{array}$ & $\begin{array}{l}21 / 19 / 8 / \\
22\end{array}$ & $\begin{array}{l}30 / 19 / \\
15 / 6\end{array}$ & 0.647 & 0.039 & & 0.043 & 2.1 (1.0 to 4.3 ) & & \\
\hline
\end{tabular}

Continuous values are shown as mean \pm SD. Categorical variables are expressed as numbers SMD Sternomental distance

${ }^{a}$ Comparisons of baseline (before the intervention) mouth aperture, SMD, and Mallampati score between the two groups using Student's t-test (mouth aperture and SMD) and linear-by-linear association (Mallampati score)

${ }^{b}$ Comparisons of mouth aperture, SMD, and Mallampati score after the intervention, between the two groups using Student's t-test (mouth aperture and SMD) and linear-by-linear association (Mallampati score)

${ }^{c}$ Comparison of the incidence of Mallampati score 1 after intervention between the two groups using chi-square test

${ }^{d}$ Comparisons of mouth aperture and SMD between before and after the intervention within the exercise group using paired t-test 
Table 3 Intubation difficulty, intubation time, and soft tissue injury

\begin{tabular}{|c|c|c|c|c|c|}
\hline & $\begin{array}{l}\text { Control }(n= \\
68)\end{array}$ & $\begin{array}{l}\text { Exercise }(n= \\
70)\end{array}$ & $P^{a}$ & $\begin{array}{l}\text { Estimated difference }(95 \% \\
\mathrm{Cl})\end{array}$ & $\begin{array}{l}\text { Odds ratio( } 95 \% \\
\text { Cl) }\end{array}$ \\
\hline Number of attempts & $1.1 \pm 0.4$ & $1.1 \pm 0.2$ & 0.253 & $0.1(-0.0$ to 0.2$)$ & \\
\hline Additional operator & $0(0 \%)$ & $0(0 \%)$ & & & \\
\hline Alternative techniques required & $8(11.8 \%)$ & $4(5.7 \%)$ & 0.207 & & 0.5 (0.1 to 1.6$)$ \\
\hline Cormack-Lehane grade (I/I//IIa/IIIb) & $42 / 20 / 2 / 4$ & $45 / 19 / 3 / 3$ & 0.746 & & \\
\hline Increased lifting force & $28(41.2 \%)$ & $17(24.3 \%)$ & 0.034 & & $0.5(0.2$ to 1.0$)$ \\
\hline Laryngeal pressure required & $24(35.3 \%)$ & $13(18.6 \%)$ & 0.027 & & $0.4(0.2$ to 0.9$)$ \\
\hline Vocal cord mobility (adduction) & $2(2.9 \%)$ & $0(0 \%)$ & 0.241 & & \\
\hline IDS & $1.6 \pm 2.1$ & $1.0 \pm 1.7$ & 0.086 & $0.6(-0.1$ to 1.2$)$ & \\
\hline $\begin{array}{l}\text { IDS group (easy/slight difficulty/moderate to major } \\
\text { difficulty) }\end{array}$ & $31 / 31 / 6$ & $44 / 23 / 3$ & 0.112 & & \\
\hline Intubation time (s) & $18.8 \pm 23.1$ & $12.5 \pm 5.4$ & 0.032 & $6.3(0.5$ to 12.0$)$ & \\
\hline Soft tissue injury & $14(20.6 \%)$ & $4(5.7 \%)$ & 0.009 & & 0.2 (0.1 to 0.8$)$ \\
\hline
\end{tabular}

enhanced Mallampati scores, as we had anticipated (Table 2). Strictly speaking, the mouth opening and SMD evaluated in our study are induced by active muscle contraction, and therefore, are different from the passive processes during the orotracheal intubation using direct laryngoscopy. However, improvement of joint flexibility and muscle stiffness by the exercise therapy led to a substantial treatment effect on the measured values (Table 2). The objective increase of mouth opening and neck extension seems to have influenced the clinical differences between the two groups (Table 3).

The IDS comprehensively scores intubation difficulty, because it combines 7 measurable variables (Fig. 2) [2, 22, 34]. In our study, the incidences of increased lifting force and laryngeal compression were lower in the exercise group than in the control group (Table 3). The enhanced joint range of motion and reduced muscle stiffness seem to induce the discrepancies. Conversely, the Cormack-Lehane grade did not differ between the two study groups. This suggests that comparable visualization could be obtained by increasing lifting force or laryngeal compression in the control group. The total IDS scores were also indistinguishable between the two groups (Table 3). However, the additional force and pressure necessary for patients in the control group increased intubation time and caused more frequent oropharyngeal soft tissue injury (Table 3). For the patients with a baseline Mallampati score III or IV, the pre-anesthetic intervention was associated with less intubation difficulty during anesthetic induction, which highlighted the value of the brief regimen (Table 4). The exercise intervention in our protocol was intended to increase the range of motion in jaw and neck joints by enhancing joint flexibility and reducing muscle stiffness, which seemed to be more effective for improving intubation conditions in the patients who had a relatively small mouth aperture size.

In this study, we excluded patients with temporomandibular joint disorders or cervical spine diseases. Unless active movement is impossible or would induce neurologic symptoms, patients at high risk for intubation difficulties should be able to manage the exercise and might benefit from this therapy, which would be clinically valuable. However, since this study was the first clinical trial demonstrating the effect of pre-anesthetic exercise, we excluded those patients to avoid conditions where proper stretching was not possible due to joint pain, and to maintain uniformity in the $5 \mathrm{~min}$ intervention. Longtime and steady exercise program might be more helpful

Table 4 Intubation difficulty among the patients with baseline Mallampati score III or IV

\begin{tabular}{llll}
\hline & Control $(n=28)$ & Exercise $(n=30)$ & $P^{\text {a }}$ \\
\hline Cormack-Lehane grade (I/I/IIIa/IIIb) & $11 / 12 / 2 / 3$ & $19 / 9 / 0 / 2$ & 0.098 \\
IDS group (easy/slight difficulty/moderate to major difficulty) & $8 / 15 / 5$ & $19 / 9 / 2$ & 0.029 \\
\hline
\end{tabular}

Categorical variables are expressed as patient numbers

IDS Intubation difficulty scale

${ }^{a}$ Comparisons between the two groups using linear-by-linear association (Cormack-Lehane grade) and chi-square test (IDS group) 
for this cohort. Further studies would be necessary to identify the effect of preoperative exercise regimen on intubation conditions in patients with an increased risk for difficult intubation.

Some limitations of this study should be mentioned. First, the IDS incorporates some subjective parameters: the perception of lifting force and the application of alternative techniques or laryngeal compression may vary among anesthesiologists. It was also proposed that the intubation difficulty should be evaluated with simple and objective parameters such as Cormack-Lehane grade and intubation time, rather than IDS [35]. However, in our protocol, the orotracheal intubation was performed by a single blinded anesthesiologist in each hospital to exclude any possible inter-assessor bias and increase the reliability of the IDS scoring. Furthermore, there was a significant difference in the intubation time between the control group and the exercise group. However, interrater variability still existed between the two hospitals. To minimize the bias, each investigator tried to perform every measurement or intervention uniformly and strictly according to the study protocol standardized in advance. Second, the duration from the end of exercise regimen to the anesthetic induction was not controlled uniformly. In order to maintain the blindness, the intervention was performed at the reception, not in the OR. The time for transportation to the operating room and preparation for general anesthesia was not strictly constant. However, the delay did not extend beyond $5 \mathrm{~min}$ in both hospitals, and the exercise significantly improved mouth opening and neck extension during anesthetic induction. In a previous report, simple stretching for less than 2 min alleviated passive hamstring stiffness for 20 min [12]. Third, Mallampati score, the primary outcome of the study, has been criticized for its low predictive value for difficult intubation [36-38]. However, we did not hypothesize that the pre-anesthetic regimen would reduce intubation difficulty, rather, we anticipated that the exercise therapy we describe would increase range of motions of mouth opening and neck mobility during the anesthetic induction, thereby possibly reducing intubation time and tissue injuries associated with intubation (Table 3). We utilized the Mallampati score to assess the clinical effects of the exercises over other simple lengths. Fourth, penlight examination might miss injuries in the deep hypopharyngeal or laryngeal structures. Although the appearance of bloody secretions in the oropharyngeal suction could help identify the injuries, this was also not an objective and accurate measurement. To reduce inter-rater variability and to maintain consistency of the test, a single blinded anesthesiologist in each hospital performed the examinations. Fifth, patients in our study showed a higher proportion of Mallampati score IV, compared to previous studies [39, 40]. No phonation during the measurement and the relatively small number of patients might have influenced the distribution of our findings. However, in previous research, the distribution of Mallampti scores also varied according to the patients' characteristics [41]; the most important outcome of this study was that the exercise regimen significantly changed it.

\section{Conclusion}

A 5 min pre-anesthetic exercise session facilitated mouth opening and neck extension during orotracheal intubation, improving intubation conditions and enabling faster intubation with less injury to oropharyngeal soft tissue. The clinical effect of the brief regimen seemed to be more significant in patients with higher Mallampati scores. Our results suggest that incorporation of such therapy into pre-operative procedures may be beneficial for patients. Patients undergoing general anesthesia can easily perform the simple exercise during the waiting time before anesthetic induction.

\section{Abbreviations \\ ASA: American Society of Anesthesiologists; ISD: Intubation difficulty scale (IDS); SMD: Sternomental distance

\begin{abstract}
Acknowledgements
The authors appreciate Dr. Ji Hun Jo, Pf. Jin-Hee Kim, and Pf. Jung-Hee Ryu of Seoul National University Bundang Hospital for their collecting and analyzing the data. Also, the authors thank Sunkyu Choi, MS, Medical Research Collaborating Center, Seoul National University Bundang Hospital, for his
\end{abstract} assistance in the statistical analysis.}

\section{Authors' contributions}

Study design/planning, SYL, JWP; Study conduct, SIB, SHD, JWP; Data analysis, SYL, SIB, JWP; Data interpretation, JTS, JWP; Drafting of manuscript, SYL, SIB; Revising of manuscript: SHD, JTS, JWP. All authors have read and approved the manuscript.

\section{Funding}

This study did not receive any extramural funding.

\section{Availability of data and materials}

The dataset generated and analyzed during the current study is available from the corresponding author on reasonable request.

\section{Ethics approval and consent to participate}

The study protocol was approved by the Institutional Review Boards of Seoul National University Bundang Hospital (study number: B-1709-423-307) and Jinju Gyeongsang National University Hospital (study number: GNUH 201805-019-001). Written informed consent was obtained from all patients before surgery. The study was registered at the Clinical Research Information Service (https://cris.nih.go.kr; registration number: KCT0002618; date of registration: December 28, 2017)

\section{Consent for publication}

Not applicable

\section{Competing interests}

The authors declare that they have no competing interests.

\section{Author details}

'Department of Anesthesiology and Pain Medicine, ThanQ Seoul

Thyroid-Head \& Neck Surgery Center, Seoul, South Korea. ${ }^{2}$ Department of Anesthesiology and Pain Medicine, Jinju Gyeongsang National University Hospital, Jinju, South Korea. ${ }^{3}$ Department of Anesthesiology and Pain 
Medicine, Seoul National University Bundang Hospital, 82 Gumi-ro, 173 Beon-gil, Bundang-gu, Seongnam, Gyeonggi-do 13620, Republic of Korea. ${ }^{4}$ Department of Anesthesiology and Pain Medicine, Seoul National University College of Medicine, Seoul, South Korea. ${ }^{5}$ Department of Anesthesiology and Pain Medicine, Gyeongsang National University College of Medicine, Institute of Health Sciences, Gyeongsang National University, Jinju, South Korea.

\section{Received: 13 November 2019 Accepted: 15 January 2020}

\section{Published online: 29 January 2020}

\section{References}

1. Calder I, Picard J, Chapman M, O'Sullivan C, Crockard HA. Mouth opening: a new angle. Anesthesiology. 2003;99(4):799-801.

2. Seo SH, Lee JG, Yu SB, Kim DS, Ryu SJ, Kim KH. Predictors of difficult intubation defined by the intubation difficulty scale (IDS): predictive value of 7 airway assessment factors. Korean J Anesthesiol. 2012;63(6):491-7.

3. Shiga T, Wajima $Z$, Inoue T, Sakamoto A. Predicting difficult intubation in apparently normal patients: a meta-analysis of bedside screening test performance. Anesthesiology. 2005;103(2):429-37.

4. Carlson JN, Hostler D, Guyette FX, Pinchalk M, Martin-Gill C. Derivation and validation of the Prehospital difficult airway IdentificationTool (PreDAIT): a predictive model for difficult intubation. West J Emerg Med. 2017;18(4):66272.

5. Eberhart LH, Arndt C, Aust HJ, Kranke P, Zoremba M, Morin A. A simplified risk score to predict difficult intubation: development and prospective evaluation in 3763 patients. Eur J Anaesthesiol. 2010;27(11):935-40.

6. Hirabayashi $Y$, Seo N. Use of a new videolaryngoscope (airway scope) in the management of difficult airway. J Anesth. 2007;21(3):445-6.

7. Wong DT, Mehta A, Tam AD, Yau B, Wong J. A survey of Canadian anesthesiologists' preferences in difficult intubation and "cannot intubate, cannot ventilate" situations. Can J Anesth. 2014;61(8):717-26.

8. Roth D, Pace NL, Lee A, Hovhannisyan K, Warenits AM, Arrich J, Herkner H. Bedside tests for predicting difficult airways: an abridged Cochrane diagnostic test accuracy systematic review. Anaesthesia. 2019;74(7):915-28.

9. Mourao J, Moreira J, Barbosa J, Carvalho J, Tavares J. Soft tissue injuries after direct laryngoscopy. J Clin Anesth. 2015;27(8):668-71.

10. Ghabash MB, Matta MS, Mehanna CB. Prevention of dental trauma during endotracheal intubation. Anesth Analg. 1997;84(1):230-1.

11. Michelotti A, de Wijer A, Steenks M, Farella M. Home-exercise regimes for the management of non-specific temporomandibular disorders. J Ora Rehabil. 2005;32(11):779-85.

12. Whatman C, Knappstein A, Hume P. Acute changes in passive stiffness and range of motion post-stretching. Phys Ther Sport. 2006;7(4):195-200.

13. Cronin J, Nash M, Whatman C. The acute effects of hamstring stretching and vibration on dynamic knee joint range of motion and jump performance. Phys Ther Sport. 2008;9(2):89-96

14. Halbertsma JP, van Bolhuis Al, Goeken LN. Sport stretching: effect on passive muscle stiffness of short hamstrings. Arch Phys Med Rehabil. 1996; 77(7):688-92.

15. Eriksson Crommert M, Lacourpaille L, Heales LJ, Tucker K, Hug F. Massage induces an immediate, albeit short-term, reduction in muscle stiffness. Scand J Med Sci Sports. 2015;25(5):e490-6.

16. Weerapong P, Hume PA, Kolt GS. The mechanisms of massage and effects on performance, muscle recovery and injury prevention. Sports Med. 2005; 35(3):235-56.

17. Wiktorsson-Moller M, Oberg B, Ekstrand J, Gillquist J. Effects of warming up, massage, and stretching on range of motion and muscle strength in the lower extremity. Am J Sports Med. 1983;11(4):249-52.

18. Taniguchi K, Shinohara M, Nozaki S, Katayose M. Acute decrease in the stiffness of resting muscle belly due to static stretching. Scand J Med Sci Spor. 2015;25(1):32-40.

19. Samsoon GL, Young JR. Difficult tracheal intubation: a retrospective study. Anaesthesia. 1987;42(5):487-90

20. Takahata O, Kubota M, Mamiya K, Akama Y, Nozaka T, Matsumoto H, Ogawa $H$. The efficacy of the "BURP" maneuver during a difficult laryngoscopy. Anesth Analg. 1997;84(2):419-21.

21. Frerk C, Mitchell VS, MCNarry AF, Mendonca C, Bhagrath R, Patel A, O'Sullivan EP, Woodall NM, Ahmad I. Difficult airway society intubation guidelines working g: difficult airway society 2015 guidelines for management of unanticipated difficult intubation in adults. Br J Anaesth. 2015;115(6):827-48.
22. Adnet F, Borron SW, Racine SX, Clemessy JL, Fournier JL, Plaisance $P$, Lapandry C. The intubation difficulty scale (IDS): proposal and evaluation of a new score characterizing the complexity of endotracheal intubation. Anesthesiology. 1997;87(6):1290-7.

23. Eshraghi Y, Nasr V, Parra-Sanchez I, Van Duren A, Botham M, Santoscoy T, Sessler DI. An evaluation of a zero-heat-flux cutaneous thermometer in cardiac surgical patients. Anesth Analg. 2014;119(3):543-9.

24. Mallampati SR, Gatt SP, Gugino LD, Desai SP, Waraksa B, Freiberger D, Liu PL. A clinical sign to predict difficult tracheal intubation: a prospective study. Can Anaesth Soc J. 1985;32(4):429-34.

25. Aoki O, Tsumura N, Kimura A, Okuyama S, Takikawa S, Hirata S. Home stretching exercise is effective for improving knee range of motion and gait in patients with knee osteoarthritis. J Phys Ther Sci. 2009;21(2):113-9.

26. Bennell $\mathrm{KL}$, Hinman RS. A review of the clinical evidence for exercise in osteoarthritis of the hip and knee. J Sci Med Sport. 2011;14(1):4-9.

27. Minor MA, Hewett JE, Webel RR, Anderson SK, Kay DR. Efficacy of physical conditioning exercise in patients with rheumatoid arthritis and osteoarthritis. Arthritis Rheum. 1989;32(11):1396-405.

28. Leivseth $\mathrm{G}$, Torstensson J, Reikeras $\mathrm{O}$. Effect of passive muscle stretching in osteoarthritis of the hip. Clin Sci. 1989;76(1):113-7.

29. Goyal S, Kothari N, Chaudhary D, Verma S, Bihani P, Rodha MS. Reversal agents: do we need to administer with neuromuscular monitoring - an observational study. Indian J Anaesth. 2018;62(3):219-24.

30. Dompier TP, Denegar CR, Buckley WE, Miller SJ, Hertel J, Sebastianelli WJ. The effect of general anesthesia on passive-knee-extension range of motion. J Sport Rehabil. 2001;10(4):257-66.

31. Alnaqeeb MA, Al Zaid NS, Goldspink G. Connective tissue changes and physical properties of developing and ageing skeletal muscle. J Anat. 1984; 139(Pt 4):677-89.

32. Brandenburg JE, Eby SF, Song P, Zhao H, Brault JS, Chen S, An KN. Ultrasound elastography: the new frontier in direct measurement of muscle stiffness. Arch Phys Med Rehabil. 2014;95(11):2207-19.

33. Witzmann FA, Kim DH, Fitts RH. Hindlimb immobilization: length-tension and contractile properties of skeletal muscle. J Appl Physiol Respir Environ Exerc Physiol. 1982;53(2):335-45.

34. K Nasa V, S Kamath S. Risk factors assessment of the difficult intubation using intubation difficulty scale (IDS). J Clin Diagn Res. 2014;8(7):GC01-3.

35. Sgalambro F. Proposal of a simplified intubation difficulty scale for comparing techniques and devices. BJA: Br J Anaesth. 2016;117(eLetters Supplement). https://doi.org/10.1093/bja/el_13469.

36. Khan ZH, Kashfi A, Ebrahimkhani E. A comparison of the upper lip bite test (a simple new technique) with modified Mallampati classification in predicting difficulty in endotracheal intubation: a prospective blinded study. Anesth Analg. 2003;96(2):595-9 table of contents.

37. Oates JD, Macleod AD, Oates PD, Pearsall FJ, Howie JC, Murray GD. Comparison of two methods for predicting difficult intubation. $\mathrm{Br} J$ Anaesth 1991;66(3):305-9.

38. Adamus M, Fritscherova S, Hrabalek L, Gabrhelik T, Zapletalova J, Janout V. Mallampati test as a predictor of laryngoscopic view. Biomed Pap Med Fac Univ Palacky Olomouc Czech Repub. 2010;154(4):339-43.

39. Pinto J, Cordeiro L, Pereira C, Gama R, Fernandes HL, Assuncao J. Predicting difficult laryngoscopy using ultrasound measurement of distance from skin to epiglottis. J Crit Care. 2016;33:26-31.

40. Ambesh SP, Singh N, Rao PB, Gupta D, Singh PK, Singh U. A combination of the modified Mallampati score, thyromental distance, anatomical abnormality, and cervical mobility (M-TAC) predicts difficult laryngoscopy better than Mallampati classification. Acta Anaesthesiol Taiwanica. 2013; 51(2):58-62

41. Pilkington S, Carli F, Dakin MJ, Romney M, De Witt KA, Dore CJ, Cormack RS Increase in Mallampati score during pregnancy. Br J Anaesth. 1995;74(6): 638-42.

\section{Publisher's Note}

Springer Nature remains neutral with regard to jurisdictional claims in published maps and institutional affiliations. 